\title{
GEOMETRIC MODELLING: LESSONS LEARNED FROM THE 'STEP' STANDARD
}

\author{
Michael J. Pratt \\ LMR Systems, UK
}

\begin{abstract}
The first release of the international standard ISO 10303 (STEP) occurred in 1994. Since that time there has been much activity in translator development and in the testing of STEP data transfer in industrial applications. Meanwhile, further development of the standard is proceeding, driven to some extent by experience gained in the use of the initial 1994 release. This paper surveys some of the lessons that have been learned in using STEP, concentrating mainly on issues arising in the geometric modelling area. Suggestions are made for overcoming some of the problems encountered.
\end{abstract}

Keywords: ISO 10303, STEP, shape modelling, algebraic geometry, application programming interfaces, geometric accuracy problem, procedural modelling.

\section{INTRODUCTION TO ISO 10303}

The international standard ISO 10303 (ISO 1994, Owen 1997), informally known as STEP (STandard for the Exchange of Product model data), has a very ambitious coverage: the capture, in electronic form, for data sharing and exchange, of product life-cycle data for manufactured products in general. Since the initial release in 1994 of twelve parts of ISO 10303, some thirty further parts have been published, and many more are under development.

Compared with its predecessor IGES (USPro 1996), STEP is intended to handle a much wider range of product-related data, and it is defined in terms of EXPRESS, an information modeling language that forms part of the 
standard. EXPRESS is used for the formal definition of exchangeable constructs, though the actual physical format in which the information is represented in an exchange file or a shared database is not that of EXPRESS itself. Mappings are defined between EXPRESS and the formats used in these two situations.

As it currently exists, ISO 10303 is oriented mainly towards the exchange of detailed design data. The parts of the standard that specify the semantic content of exchangeable data are called Application Protocols (APs), the currently standardized APs being as follows:

- AP 201 - Explicit draughting (1994)

- AP 202 - Associative draughting (1996)

- AP 203 - Configuration-controlled design (1994)

- AP 207 - Sheet metal die planning and design (1999)

- AP 214 - Core data for automotive mechanical design processes (2001)

- AP 224-Mechanical parts definition for process planning using machining features (1999)

- AP 225 - Structural building elements using explicit shape representation (1999)

All major CAD vendors are now marketing translators conforming to AP203, the most widely used STEP Application Protocol. Various other 'third-party' software developers also have translators available. There is an increasing number of 'success stories' concerning the productive industrial use of STEP transfers (PDES Inc. 2001). Translator development for other Application Protocols, including AP202, AP210 and AP214, is well advanced in some organizations.

The following partial list of further APs under development shows the full range of products addressed at present, and also some of the product lifecycle stages, other than detail design, that are beginning to be addressed (schematic design, engineering change management, packaging, analysis, process planning, inspection, assembly,...). Some entries on the list, for example APs 209, 210, 212 and 227, are very close to being published as new parts of the ISO 10303 standard at the time of writing.

- AP 208 - Life cycle management: change process

- AP 209 - Composite and metallic structural analysis and related design

- AP 210 - Electronic assembly, interconnection and packaging design

- AP 212 - Electrotechnical design and installation

- AP 213 - NC process plans for machined parts 
- AP 216 - Ship moulded forms

- AP 217 - Ship piping

- AP 218 - Ship structures

- AP 219-Dimensional inspection

- AP 220 - Process planning, manufacture and assembly of layered electronic products

- AP 221 - Process plant functional data and its schematic representation

- AP 222 - Design and manufacturing data for composite structures

- AP 223 - Design and manufacturing data for cast parts

- AP 227 - Plant spatial configuration

- AP 228 - Building services: HVAC

- AP 233 - Systems engineering data representation

- AP 235 - Materials information for design and verification of products

- AP 236 - Furniture product and project data

Between the inception of work on ISO 10303 in 1984 and its first release in 1994, the architecture of the standard evolved significantly as the scale of the undertaking expanded. This evolution is still continuing, for several reasons:

- The pace of development is slow under the present mechanisms;

- Problems of scale have not yet been fully solved;

- Requirements are emerging for

- interoperability between different STEP APs;

- interoperability between ISO 10303 and other related standards, notably UML and XML;

- new modes of accessing the data.

The first two of these, and the AP interoperability requirement, are being addressed by introducing a new modular architecture. In future, APs will be composed of separately validated shareable modules covering self-contained aspects of product modeling. AP interoperability will be enhanced through the sharing of common modules. The last two items are being separately addressed.

However, this paper is not primarily concerned with these high-level developments to the standard. The intention here is to review some of the experience gained in the early use of the standard, with particular regard to its shape modeling aspects. The following sections will also outline some of the measures being taken to improve the success of product model exchanges, and will suggest some possible future developments that may enhance the use of STEP. 


\section{IMPROVEMENTS IN ISO 10303 SHAPE MODELING}

The basic shape modelling resource of ISO 10303 is Part 42 of the standard (ISO 1999), 'Geometric and topological representation'. It provides representations of the wireframe, surface and boundary representation solid types, together with constructive solid geometry (CSG) capabilities (Hoffmann 1989). All the curve and surface types commonly implemented in CAD systems are available. This resource formed part of the initial 1994 release of the standard, but was updated in 1999. The major change was the provision of a wider range of primitives for use in CSG, as requested by AP developers for the building and construction industries. Additionally, a completely new surface type was included, the Dupin cyclide (see Section 4.1), which has applications in blending and in the design of ducting in heating and ventilation systems.

More major changes in ISO 10303 geometric modelling capability will result from the work of the ISO TC184/SC4 Parametrics Group, who are working to add new capability for the transmission of CAD models with parametrization, geometric constraints and features. This activity is discussed in some detail in Section 4 of the paper.

\section{THE GEOMETRIC ACCURACY PROBLEM}

Experience with the use of AP203 has shown that STEP is capable of transmitting pure geometric models with a good degree of success, but has also revealed one major difficulty that prevents STEP from complete fulfilment of its intended potential. This is not a flaw in the standard itself, but is related to implementational differences in CAD and other product realization systems. It is known as the geometric accuracy problem; see Hoffmann (1989) for a good discussion.

Such accuracy concerns arise because Brep shape models as used in CAD systems contain two classes of information, topological and geometrical. It is impossible to maintain complete consistency between these two aspects of the model because of the occurrence of computational errors in geometric calculations. Unfortunately, inconsistency problems also often occur as a result of inappropriate modelling practice by system users. As an example of such an inconsistency, consider a case where the model topology states that two edges in the model are connected, sharing a common vertex, while the geometry says that the end-points of the edges in fact have slightly different coordinates. In all CAD systems there is an interpretation of 'slightly' in the 
last sentence in terms of one or more geometric accuracy parameters. These are used to resolve questions of coincidence or codirectionality. The trouble is that the various CAD systems are not compatible in how they approach this problem. For example, one widely-used system judges points to be coincident if they are within $10^{-4}$ unit of each other, while another uses $10^{-7}$ unit for the same criterion. Thus geometric elements that are 'connected' under the accuracy standard of the first system are almost certainly not connected according to the second. The topology transmitted in the STEP model will say that they are connected, but the geometry as interpreted by the receiving system will disagree, and the overall model exchange will fail.

Three main approaches have been suggested for overcoming the geometric accuracy problem. These are discussed below.

\subsection{Model Quality Testing}

Tests have revealed that the geometric accuracy problem is severely exacerbated by the fact that many CAD systems generate models with undesirable characteristics. These are often the result of parametric changes to the model; they include such phenomena as very small faces and very short edges in the model. Often these details are so small that they cannot be seen on the CAD system screen without strong magnification. From the engineering point of view they are totally negligible, but in data exchange they give rise to many situations where the geometric accuracy problem arises. Some CAD system vendors have already implemented model checking procedures so that undesirable model characteristics can be detected and eliminated before a STEP exchange file is generated. However, although the use of such methods avoids a significant proportion of the cases where the geometric accuracy problem would otherwise arise, it certainly does not completely eliminate it.

\section{2 'Model healing'}

This approach is based on the intelligent detection and classification of individual geometric accuracy problems. According to the nature of a particular occurrence it may be possible to make slight adjustments to the geometry of an edge or a face to remove the detected anomaly. Although this approach has had some limited success it is generally regarded as being rather dangerous. A geometric change that removes a local problem in one place on the model may well create one or more further local problems elsewhere. 


\subsection{Semantic study}

The most fundamental approach takes the view that at present the nature of the geometric accuracy problem is not well understood. The semantics of such phrases as 'coincident to within system tolerances' have to be carefully worked out in the context of three-dimensional shape modeling, and agreement on those semantics must be obtained amongst CAD system vendors so that a more uniform approach is taken in their various products.

The kind of situation that needs to be addressed is illustrated by the case of three collinear points, A, B and C. A may be close enough to B for the system to judge the two to be coincident. The same may be true of $B$ and $C$. However, coincidence of $\mathrm{A}$ with $\mathrm{B}$ and of $\mathrm{B}$ with $\mathrm{C}$ does not necessarily imply that $\mathrm{A}$ is coincident with $\mathrm{C}$, since the actual distance between these points may lie outside the system's 'point coincidence' tolerance.

\subsection{Alternative Approaches}

There are other longer-term possibilities for alleviating or overcoming the geometric accuracy problem that have not so far been addressed. The first is simply to improve the training given to CAD system operators. At present, training is usually concentrated on presentation of the functionality available in a system. What is needed is further instruction on model creation strategy - how to use that functionality to the best advantage, and in particular how to ensure good model quality. This is a whole subject in its own right, and attention to this matter could save industry a great deal of money that is currently expended in cleaning up CAD models for use in downstream applications. However, training issues are outside the scope of the present proceedings, and will not further be explored here. The following sections are devoted to a discussion of three other possible approaches to the achievement of greater robustness in CAD modeling.

\subsubsection{Use of algebraic geometry representations}

The current de facto standardization on the use of NURBS for free-form geometry is not universally applauded. Although in principle NURBS provide a useful general-purpose representation encompassing both 'simple' and free-form geometry, they are acknowledged to have some disadvantages. These include

- Problems with automatically generated parameterizations 
- Propagation of multiple knots on surface boundaries into the surface interior as crease lines

- The difficulty of determining surface intersection curves in a way that is not only fast and accurate but also guarantees to find all branches of the intersection (Pratt and Geisow 1986).

CAD systems in current use employ surface representations of parametric degree up to $20 \times 20$. Since a degree $n \times n$ surface patch has $(n+1)^{2}$ control points, each with three degrees of freedom in the polynomial case and four in the rational case, the number of degrees of freedom in a rational $20 \times 20$ patch is 1764. Composite NURBS or Bézier surfaces are of course made up of meshes of patches. The question is, do we really need all these degrees of freedom for design purposes, or are they in fact an embarrassment?

The algebraic degree of an $n \times n$ parametric surface is $\leq 2 n^{2}$, and the intersection of two such surfaces has algebraic degree $\leq 4 n^{4}$. The geometric interpretation of this is that there can be as many as $4 n^{4}$ intersections of the curve with a plane. In purely qualitative terms, this implies that the intersection curve can be highly oscillatory with respect to a given plane, and one manifestation of this is that it may comprise many disjoint branches. Some of these may be small loops, difficult to detect using conventional 'marching' strategies for computing intersection curves (Pratt and Geisow 1986). However, failure to detect them, or to detect limiting points such as cusps, self-intersections or self-tangencies of the curve, may lead to incorrect topological structures in a $\mathrm{CAD}$ model and consequent inconsistency of geometry and topology.

One possible method of overcoming these problems is to find a way of modeling with low-degree algebraic rather than parametric surfaces. This would avoid at least some of the problems associated with the use of geometric elements possessing far more degrees of freedom than are required for most engineering purposes.

Several authors have discussed the virtues of using low-degree algebraic surfaces for geometric modelling. The most appropriate degree to use is still an open question. A compromise is needed between geometric flexibility for the modelling of general shapes and ease of control for the designer. The primary advantage of the parametric approach to surface representation lies in the availability of control point formulations providing at least a semiintuitive means of shape control; such control is proving less easy to provide for modelling with algebraic surfaces. 
It is generally considered that the general quadrics provide insufficient freedom for free-form design, though there has recently been some work by Froumentin et al. on their use in computer graphics (Froumentin and Chaillou 1997). The cubic surfaces have been investigated by Sederberg (1990), who has suggested a method for their use in the construction of piecewise-defined free-form shapes. However, this method is not intuitive for the nonmathematical designer, and problems can arise with unexpected changes in surface topology as defining parameters are varied.

While the general cubic possesses 19 degrees of freedom the general quartic has $34^{1}$, which poses an even greater problem in finding means for its intuitive control. For this reason investigation of the quartics for design has been restricted to special cases, notably the Steiner surfaces (Sederberg and Anderson 1985) and the Dupin cyclides (Dutta, Martin and Pratt 1993). The latter are easy to handle from the designer's point of view, and have been found useful for the construction of blend surfaces (Boehm 1990, Pratt 1990, Pratt 1995), though Martin, de Pont and Sharrock (1986) found that they give insufficient freedom for fully free-form design. For this reason Degen and Pratt have been studying supercyclides, a class of quartics initially identified by Degen $(1982,1986)$, which proves to contain projective transformations of the Dupin cyclides and therefore permits greater freedom for shape modelling. These surfaces can can be parameterized by a conjugate net of conic curves (Degen 1994, Pratt 1996, Pratt 1997), which are all circles in the special case of the Dupin cyclide. The supercyclides have great potential as flexible blending surfaces in CAGD, and also have other desirable properties as described by Pratt (1997). Some work in supercyclide blending has reported by Degen (1994) and by Allen and Dutta (1996).

It is possible that supercyclides, unlike the Dupin cyclides, will provide sufficient geometric freedom for use in fully free-form surface design. In this context they have the advantage of naturally giving rise to four-sided patches, as used in existing practical CAD systems, rather than three-sided patches as generated by most other approaches to algebraic surface modelling (Bajaj 1993, Dahmen and Thamm-Schaar 1993, Sederberg 1985). There is no known work at present on free-form surface design using supercyclides, though this is likely be a fruitful area of research.

Apart from the Steiner surfaces, the Dupin cyclides and the supercyclides, one other class of quartic surfaces has been suggested for possible use in CAGD. This is the class of generalized cyclides extensively studied by

${ }^{1}$ The implicit equation of a surface of algebraic degree $n$ contains $1 / 6(n+1)(n+2)(n+3)$ coefficients, of which one may be chosen arbitrarily 
Darboux (1896), which have recently arisen in some work by Paluszny and Boehm (1998).

Besides the work already cited, there has been extensive study of the use of algebraic surfaces purely for blending purposes (see Rockwood 1997 and the references therein). However, the intention of this section has been to highlight research that may lead to a more general shape modeling capability using algebraic surfaces, with consequent improvements in accuracy and robustness resulting from the elimination of the unwarranted degrees of freedom associated with parametric geometry.

\subsubsection{Use of procedural representations}

A further possibility for overcoming the geometric accuracy problem is to transfer a procedural rather than an explicit description of the shape model. If the shape is described purely in terms of the sequence of modelling operations used to construct it, then these operations will be performed in the receiving system following a transfer, and this will lessen the possibility of conflict between the internal geometric tolerances of the sending and receiving systems.

Most CAD systems use a hybrid representation in which, for example, a 2D profile may be represented explicitly in terms of edges and vertices and a solid of extrusion derived from it through the use of an extrusion operator. In such a case the explicitly represented profile could still give rise to accuracyrelated inconsistency in a model exchange. However, such problems are usually much less severe for curves and points in a $2 \mathrm{D}$ context than they are for surface intersections in 3D. It is believed that the use of a procedural approach will allow most such 3D computations to be performed in the receiving system, thus circumventing the accuracy problem. Work is already in progress on the STEP exchange of procedural models, and further details are given in the next section of the paper.

\subsubsection{Use of new forms of representation}

It has been suggested that the perceived problems of geometry/topology inconsistency, and its manifestation the geometric accuracy problem, can best be solved through the use of a single self-consistent model representation. Rossignac and O'Connor (1990) have proposed the use of Selective Geometric Complexes (SGC), and Rossignac (1997) has more recently extended this idea to Structured Topological Complexes (STC). Meanwhile Middleditch et al. (2000) are developing a similar but more general approach 
in the context of the Djinn API work in the UK (Armstrong et al. 2000). These are set-theoretic approaches. Like their forerunner, CSG, they suffer from the problem that they depend on the underlying concept of the neighbourhood of a point. They are therefore difficult to implement since no convincing way has yet been found of handling neighbourhoods computationally. However, there may be some hope here for the future if the implementational difficulties can be overcome.

\section{THE 'DESIGN INTENT' PROBLEM}

The types of geometric models that can currently be exchanged by STEP are 2D drawings, wireframes, surface models and solid models of the CSG and Brep types (including faceted Brep models). This reflects the state of CAD shape modelling technology as it was in the mid-1980s when STEP development commenced. Modern CAD systems generate feature-based models that employ parameterization and constraints to capture aspects of what is known as 'design intent'. In particular, this information captures knowledge about how the model may or may not be edited (e.g., for purposes of design optimization). Parameterization represents design freedom; conversely, constraints represent design restraint, often imposed in the interests of preserving functionality if design changes are made. Both parameterization and constraints are routinely applied to design features and their relationships in the model.

At present, ISO 10303 cannot capture or transfer any of the design intent information mentioned in the last paragraph. The result is that the 'intelligent model' generated in the sending system becomes a 'dumb model' in the course of translation between systems, simply because the additional behavioural information is lost. Thus in the receiving system, following a model transfer, no knowledge is available about how the model may be edited for subsequent applications. A few commercial systems can automatically reconstruct some of the lost data, particularly regarding features, but for the most part this has to be a time-consuming manual process requiring a good deal of guesswork.

There are two possible complementary approaches to the capture and transmission of design intent in a CAD model. The first is to supplement the existing Brep type of model transfer with parametrization and constraint information that specifies relationships between geometric elements of the model. This will allow some degree of intelligent editing following transfer of the model. The additional data are redundant at the time of model 
exchange, since they only assert relationships that already exist in the model. However, following the transfer these relationships are required to be maintained in the receiving system, so that some edits are valid while others are not.

A more radical approach is to transfer the model in terms of its constructional history, i.e., the sequence of operations used to build it. This clearly captures the design intent more closely, and it is also closer to the way that most CAD systems represent their model data internally. In fact, the Brep models transferred by STEP today are really subsidiary models generated by the systems for purposes of display, geometric calculation and user interaction.

Work is in progress on both these approaches to the enhancement of the STEP shape modelling capability. Both are required in practice, because the procedural or constructional history approach often makes reference to explicit elements that may have imposed parameterization and constraints. An example is the use of a $2 \mathrm{D}$ sketch or profile, made up of explicit curve segments, in the generation of a procedurally defined composite surface by the use of a linear or rotational sweep operation. The current status of this work is reviewed below.

\subsection{Parameterization and constraints in explicit STEP models}

A new STEP resource, Part 108 of the standard, is currently being developed. This is upwardly compatible with previously published STEP capabilities in the sense that it requires no changes to them to provide its additional functionality. This document contains five schemas that provide for the capture and transfer of the following types of information:

- Parameters associated with attributes (in particular, dimensional attributes) in an explicit shape model.

- Constraints expressed as explicit mathematical relationships between parameters.

- Geometric constraints expressed descriptively (including, for example, parallelism, perpendicularity and tangency between geometric elements).

- Further specializations of the geometric constraints for use in a 3D assembly modelling context.

- Sketches or profiles, composed of curve elements, for use in constructional operations involving extrusions, rotations, etc. 
The geometric constraints defined in the third schema are implemented in all major CAD systems. Because their semantics are universally understood, these relationships are specified descriptively rather than analytically. Sending and receiving systems are expected to formulate them mathematically in whatever way is most convenient for their internal algorithms.

At the time of writing, the current version of the Part 108 document (ISO 2001 ) is being prepared for the first stage of the formal process of acceptance as a published part of the STEP standard.

\subsection{Transfer of construction history models in STEP}

ISO 10303 in its current form makes no provision for the exchange of procedurally defined or constructional history models, except for a limited CSG capability. However, work has now started on the development of new capabilities for this purpose. This work is bound to take some time, because it is a considerable departure from the 'snapshot' types of models that STEP has previously been concerned with. Initial work is primarily concerned with providing a proof-of-concept demonstration of the transfer of a simple constructional history model. This only demands the creation of limited new resources, and if the experiment is successful these will be built upon to provide a comprehensive new mechanism for model exchange. The perceived requirements are as follows:

- Representations will be needed for constructional operations for a full range of modelling elements. This includes not only the low-level topological and geometrical elements of Brep modelling, but also higherlevel constructs such as swept volumes and other types of design features.

- A method is needed for capturing the effect of a screen pick of an element by the user of the sending system.

- A structuring method method is required, because history models are not purely sequential. In an assembly, for example, each part will have its own sequential history.

- A mechanism should be provided for the inclusion of 'design rationale' information, which explains why a particular configuration is modelled in a particular way. At present there is no means for the automatic capture of such information, and the best that can be done is to provide hooks for the association of text files with the part models.

- Query capabilities are also needed, because the precise result of a constructional operation is not always known until the operation is 
completed. An example is provided by an edge blending procedure, which may generate one or more new faces in the model, depending on the proximity of other modelling elements to the edge concerned.

The first and second entries on the list above merit further explanation.

In the case of model elements already defined by STEP, such as faces, edges, vertices and various types of curves and surfaces, the entities that represent these elements in the modelling schemas serve a dual purpose as creation operations for them. Thus, as far as these low-level requirements are concerned, much of what is needed already exists. However, most CAD system modelling today is performed in terms of high-level design features, and for most of these STEP does not currently have representations. Thus, the first major task will be to write specifications of a comprehensive range of design feature types using the ISO 10303 information modelling language EXPRESS.

The provision of this new capability in ISO 10303 will amount to the specification of a standardized applications programming interface (API) for feature-based CAD modellers. Several proposals exist for standardized CAD system APIs, and they have recently been surveyed to see whether any of them can be used as a basis for developing the new ISO 10303 resource (Pratt 1998, Pratt and Anderson 2001), but none has proved entirely suitable.

Regarding the capture of screen pick information, this is the crux of the wellknown persistent naming problem (Capoyleas, Chen and Hoffmann 1996, Kripac 1995). It has not been fully solved by any of the CAD system developers, as far as is known, but research is in progress that may provide a solution that will work in most commonly occurring cases (Raghothama and Shapiro 1997). However, it is currently thought that the problem is less severe in the context of data exchange between CAD systems than it is in updating a model internally in a single system, which is the situation usually considered. The suggested approach for data exchange is to transmit the picked element explicitly (i.e., as an explicit face, edge or vertex) in the history file. In the receiving system the rebuilding of the model from the history data will be in progress, and it will then only be necessary to compare the transmitted explicit element with elements of that type in the partially reconstructed model. Once a match is obtained the picked element is unambiguously identified in the receiving system. It is possible that experience will show the need for more sophisticated mechanisms in complex cases, but it is believed that the approach described will be sufficient for most purposes. 
The status of the constructional history work is as follows at the time of writing. Specifications of feature types for the chosen test part have been written, and the format of the construction history transfer file determined. A hand-coded transfer file for the part has been created. Several CAD vendor companies have been approached as participants in the proof-of-concept demonstration, whose objectives are (i) to generate the example transfer file directly from a CAD system, and (ii) to regenerate a parametric model in a CAD system by reading in the example file. Meanwhile, other experiments are in progress that are not currently using STEP for the intermediate transfer file. One of these is at KAIST in Korea, where constructional history model exchange between two similar systems has already been demonstrated. A second is at NIST in the USA, where a procedural model has been represented in a STEP-related format from a model in a third CAD system. When the file was read back into the same system the design intent proved to have been preserved. In both cases, it will be possible to migrate towards the use of strict STEP methodology. Once these preliminary experiments are complete, a formal ISO project will be started for the full development of a constructional history exchange specification for the STEP standard. It should also be mentioned that several commercial translator providers have written direct constructional history translators (i.e., not based on any neutral standard) between pairs of major CAD systems, but of course the technology for these is purely proprietary.

It was mentioned earlier that the use of procedural model transfer may help to overcome the geometric accuracy problem, because it largely avoids the mismatch of numerical tolerances in the sending and receiving systems. It is also believed that constructional history models are more suitable than explicit models for long-term archiving, since they are more concise and less closely related to any specific modelling technology.

\section{CONCLUSIONS}

The current status of geometric model representation in ISO 10303 (STEP) has been surveyed, and some of the problems identified in early experience with STEP data exchange described. The geometric accuracy problem was initially a serious obstacle to successful model exchange. However, now that this has to some extent been overcome, loss of 'design intent' is emerging as the primary problem in the use of transferred STEP models for downstream activities following a model exchange. Various current and future 
approaches to overcoming both the accuracy problem and the design intent problem in CAD data exchange have been reviewed.

\section{REFERENCES}

Allen, S., and Dutta, D. (1996). Supercyclides and blending, Computer Aided Geometric Design, Vol. 14, No. 7, pp. 637-651.

Armstrong, C., Bowyer, A., Cameron, S., Corney, J., Jared, G., Martin, R., Middleditch, A., Sabin, M., Salmon, J. and Woodwark, J. (2000). Djinn: Specification and Report, Information Geometers, Winchester, UK.

Bajaj, C.L. (1993). The emergence of algebraic curves and surfaces in geometric design. In Martin, R.R. (ed.): Directions in Geometric Computing, Information Geometers Ltd., Winchester, UK.

Boehm, W. (1990). On cyclides in geometric modelling, Computer Aided geometric Design, Vol. 7, Nos. $1-4$, pp. $243-255$.

Capoyleas, V., Chen, X. and Hoffmann, C.M. (1996). Generic naming in generative constraint-based design, Computer Aided Design, Vol. 28, No. 1, pp. 17 - 26.

Dahmen, W., and Thamm-Schaar, T.-M. (1993). Cubicoids: Modelling and visualization, Computer Aided Geometric Design, Vol. 10, No. 2, pp. 93-108.

Darboux, G. (1896). Sur une Classe Remarquable de Courbes et de Surfaces Algébriques, $2^{\text {nd }}$ edition, Gauthier-Villars, Paris.

Degen, W.L.F. (1982). Surfaces with a conjugate net of conics in projective space, Tensor, N.S., Vol. 39, pp. 167-172.

Degen, W.L.F. (1986). Die zweifachen Blutelschen Kegelschnittflächen, Manuscripta Mathematica, Vol. 55, pp. 9-38.

Degen, W.L.F. (1994). Generalised cyclides for use in computer aided geometric design. In Bowyer, A.D. (ed.): Computer Aided Surface Geometry and Design, pp. 349-363, Oxford University Press (Proc. 4th IMA Conf. on the Mathematics of Surfaces, Bath, UK, Sept. 1990).

Dutta, D., Martin, R.R. and Pratt, M.J. (1993). Cyclides in surface and solid modeling, IEEE Computer Graphics \& Applications, Vol. 13, No.1, pp. 53-59.

Froumentin, M. and Chaillou, C. (1997). Quadric surfaces: A survey with new results. In Goodman, T.N.T. and Martin, R.R. (eds.): The Mathematics of Surfaces VII, Information Geometers Ltd., Winchester, UK (Proc. 7th IMA Conf. on the Mathematics of Surfaces, Dundee, Scotland, Sept 1996).

Hoffmann, C.M. (1989). Geometric and Solid Modeling, Morgan Kaufmann, San Mateo, CA. ISO (1994a). ISO 10303:1994 - Industrial Automation Systems and Integration - Product Data Representation and Exchange, International Organization for Standardization, Geneva, Switzerland. (The ISO catalogue is at http://www.iso.ch/cate/cat.html; search on 10303 for a listing of parts of the standard).

ISO (1999). ISO 10303-42:1999 - Industrial Automation Systems and Integration - Product Data Representation and Exchange - Geometric and topological representation, International Organization for Standardization, Geneva, Switzerland (Edition 2 of this STEP resource part).

ISO (2001) ISO TC184/SC4/WG12 N908: Parameterization and constraints for explicit geometric product models - Working Draft of ISO 10303-108. Available at http://www.nist.gov/sc4/wg_qc/wg12/ 
Kripac, J. (1995). A mechanism for persistently naming topological entities in history-based parametric solid models. In Hoffmann, C.M. and Rossignac, J.R. (eds.): Proc. $3^{\text {rd }}$ ACM Symposium on Solid Modeling and Applications, Salt Lake City, 17 - 19 May 1995, pp. 21-30. ACM Press, New York, NY.

Martin, R.R., de Pont, J.J. and Sharrock, T.J. (1986). Cyclide surfaces in computer aided design. In Gregory, J.A. (ed.): The Mathematics of Surfaces, Oxford University Press (Proc. 1st IMA Conf. on the Mathematics of Surfaces, Manchester, UK, Sept. 1984).

Middleditch, A.E., Reade, C.M.P. and Gomes, A.J. (2000). Point-sets and cell structures relevant to computer aided design, Int. J. Shape Modeling, Vol. 6, No. 2, pp. 175-206.

Owen, J. (1997). STEP: An Introduction, $2^{\text {nd }}$ edition, Information Geometers Ltd., Winchester, UK.

Paluszny, M. and Boehm, W. (1998). General cyclides, Computer Aided Geometric Design, Vol. 15, No. 7, pp. 699-710.

PDES Inc. (2001). Web site http://pdesinc.aticorp.org/ (follow the link to 'Success stories').

Pratt, M.J. (1990). Cyclides in computer aided geometric design, Computer Aided Geometric Design, Vol. 7, Nos. 1-4, pp. 221-242.

Pratt, M.J. (1995). Cyclides in computer aided geometric design II, Computer Aided Geometric Design, Vol. 12, No. 1, pp. 1-22.

Pratt, M.J. (1996). Dupin cyclides and supercyclides. In Mullineux, G. (ed.): The Mathematics of Surfaces VI, pp. 43-66, Oxford University Press (Proc. 6th IMA Conf. on the Mathematics of Surfaces, Brunel University, Uxbridge, UK, Sept. 1994).

Pratt, M.J. (1997). Quartic supercyclides I: Basic theory, Computer Aided Geometric Design, Vol. 14, No. 7, pp. 671-692.

Pratt, M.J. (1998). Extension of the standard ISO 10303 (STEP) for the exchange of parametric and variational CAD models. In Jacucci, G., Olling, G.J., Preiss, K. and Wozny, M.J. (eds.): CD-ROM Procs. of IFIP WG5.2/5.3 conference PROLAMAT '98, Trento, Italy, Sept. 1998; Kluwer Academic Publishers, Boston, MA.

Pratt, M.J. and Anderson, B.D. (2001). A shape modelling applications programming interface for the STEP standard, Computer Aided Design, to appear.

Pratt, M.J. and Geisow, A.D. (1986). Surface/surface intersection problems. In Gregory, J.A. (ed.): The Mathematics of Surfaces, Oxford University Press (Proc. 1st IMA Conf. on the Mathematics of Surfaces, Manchester, UK, Sept. 1984).

Raghothama, S. and Shapiro, V, (1998). Boundary representation deformation in parametric solid modeling, ACM Trans. on Graphics, Vol. 17, No. 4, pp. 259-286.

Rockwood, A.P. (1997). Blending. In Bloomenthal, J. (ed.): An Introduction to Implicit Surfaces, pp. 197-221, Morgan Kaufmann, San Francisco, CA.

Rossignac, J.R. (1997). Structured topological complexes: A feature-based API for nonmanifold topologies. In Hoffmann, C.M. and Bronsvoort, W.F. (eds.): Proc. $4^{\text {th }} A C M$ Symposium on Solid Modeling and Applications, Atlanta, GA, May 1997; ACM Press, New York, NY.

Rossignac, J.R. and O'Connor, M. (1990). SGC: A dimension-independent model for pointsets with internal structures and incomplete boundaries. In Wozny, M.J., Turner, J.U. and Preiss, K. (eds.): Geometric Modeling for Product Engineering, North-Holland Publ. Co. (Proc. IFIP/NSF Workshop on Geometric Modeling, Rensselaerville, NY, Sept. 1988).

Sederberg, T.W. (1990). Techniques for cubic algebraic surfaces: Parts 1 and 2, IEEE Computer Graphics \& Applications, Vol. 10, No. 5, pp. 14-25 and Vol. 10, No. 6, pp. 12 21.

Sederberg, T.W. (1985). Piecewise algebraic surface patches, Computer Aided Geometric Design, Vol. 2, Nos. 1-3, pp, 53-60. 
Sederberg, T.W. and Anderson, D.C. (1985). Steiner surface patches, IEEE Computer Graphics \& Applications, Vol. 5, No. 5, pp. 23-36.

USPro (1996). Computer Aided Processing of Engineering Drawings and Related Documentation (IGES). US Product Data Association, Charleston, SC, USA (IGES Version 5.3: American National Standard USPRO/IPO-100-1996). 